\title{
Personalized application for multimodal route guidance for travellers
}

\author{
Maria Panou
}

Received: 10 November 2011 / Accepted: 6 December 2011 /Published online: 29 December 2011

(C) The Author(s) 2011. This article is published with open access at SpringerLink.com

\begin{abstract}
Purpose Studies about the use of internet-based services and especially those that are related to services on-the-move, reveal that their propagation is rapidly expanding, with high increase probability in the next years. It is about services that are offered through mobile devices (PDAs, mobile phones) where the travelers wish to receive geographically updated information. The present paper aims at the optimization of public transport (PT) information services that are provided on mobile devices, for travelers of PT means, through their personalization.

Methods A specific innovative algorithm is proposed along with the necessary parameters (dynamic and semi-dynamic) that support a holistic personalization. The algorithm is based on each user specific profile, based on the history of his/her previous selections. The proposed algorithm constitutes a ready service, that can be adopted by the appropriate stakeholders in cooperation with content providers such as public transport route providers and dynamic digital maps.

Results The developed algorithm was tested by 10 users. In general, it was positively evaluated by the majority of the users, with a varying acceptance and confidence levels. Users evaluated also the personalization parameters, giving to the maximum walking distance between PT means the highest score.

Conclusions The innovation and limitations of the current algorithm are discussed, followed by proposals for extensions and further research.
\end{abstract}

Keywords Personalization · Route guidance $\cdot$ Multimodal routes $\cdot$ Walking distance $\cdot$ Interchanges

\section{Panou $(\bowtie)$}

Hellenic Institute of Transport,

6th Km. Charilaou-Thermis,

57001 Thessaloniki, Greece

e-mail: mpanou@certh.gr

\section{Introduction}

There is an increasing use of the internet for information retrieve, by many users. The new market trend in the area of web-based information services concerns travelers information services. These are services that are offered through the mobile phone or other mobile devices (PDA).

The total number of mobile services users reached 5 billion, according to Ericsson, while back in 2000, around 720 million people worldwide used mobile services. Also, studies have shown that pretty soon $80 \%$ of the world's population will access the web via mobile devices. Ericsson estimates that we'll see around 50 billion devices connected to mobile networks till the end of the decade [3]. No matter how bad the world recession becomes, worldwide mobile services revenue e.g. those useful value-added services offered to customers by operators, will grow by at least $1.2 \%$ annually through to 2014 (ABI Research). Operators need to encourage customers to maintain 'nice-to-have' data services or addition of more utilitarian ones. Content downloads will be the first casualties of an extended recession [1].

A study of global mobile device behavior (with 34,000 interviews with mobile users in 43 countries), examined the activity level of various services on mobile devices from 2010 to 2011. Results showed that mobile phone users that use their devices for navigation will increase from $8 \%$ in 2010 to $13 \%$ in 2011, while those that use location services will increase to $12 \%$ in 2011 from $9 \%$ in 2010 [8]. Also, the mobile phone is the preferred mean for Navigation in all continents, except India [5]. More specifically, among the ten consumer applications to watch in 2012 include Location-based services (LBSs). Location is one of the main enablers that deliver services to users based on their context and, Gartner expects the total user base of consumer LBSs to reach 1.4 billion users by 2014. Such location-based 
services are useful not only for pedestrians but also for public transport (PT) means users and drivers. The travelers can get updated information covering a wide area of services, such as travelling/trips (optimal routes, public transport means selection, timetables info), weather information, traffic flow, etc.

Furthermore, as many as $40 \%$ of international travellers already own a smart phone with internet and e-mail access and other functions, while $57 \%$ have a conventional mobile phone ( $2 \%$ still do not have a mobile phone), this year's World Travel Monitor revealed. More than $40 \%$ of smart phone owners already use their devices to get destination information, and $34 \%$ of business travellers / $26 \%$ of leisure travellers use them to make booking changes during their trip. Moreover, the travel industry is one of the obvious sectors for use of important real-time data, such as trip planning, public transport timetables, etc. [9].

Unfortunately, the user gets generic information, which is not individualized according to his/her special needs and wants, leading to a decreased effectiveness of the service and finally to a lower acceptance rate by the users. Thus, LBS strive to deliver features and functionalities in tune with the user's context, taking into account the user's location, personal preference, gender, age, profession, intention and so on, thus offering a more-intelligent user experience than basic location services can. Gartner analysts believe contextaware services are a key trend for mobile apps, and location is a key enabler of that [2].

\section{Route selection personalization parameters}

In this section, the parameters that relate to the provision of personalized information to the travelers for routes, with any public transport mean in order to reach their destination, are presented. These parameters are divided in dynamic and semi-dynamic and refer to the route characteristics and to the user type.

The basic traveler service personalization that is described in the present article, assumes that the user wishes to get to a specific point. The system has to inform him/her on the route that will follow and the way to be transported, proposing multimodal solutions with public transport (PT) means that are according to the user's preferences, based on his/her previous selections.

\subsection{Dynamic parameters}

Dynamic parameters are those that are calculated automatically by the system and are updated according to the user's selection. Starting with the route characteristics, there are various possible priorities based on which the traveler can select the route that will follow in order to reach his/her destination. The most important according to ASK-IT project [7] follow below with a prioritisation order:

- Route with walking distance less than the maximum acceptable one by the user.

- Route with acceptable/ preferred (by the user) transportation mean types.

- Route with number of interchanges (change of transport mean) less than the maximum acceptable by the user.

- Shortest route.

- Nearest route (the one with the less possible distance from the user's starting point).

- Cheapest route.

- Accessible route (for travelers with mobility impairments).

- Most picturesque/interesting route.

\subsection{Semi-dynamic parameters}

Semi-dynamic parameters are the ones that the user sets each time he/she starts using the application, unless the relevant characteristics remain the same since he/she last used it. According to IM@GINE IT project [4] the users can be categorized based on the reason for travelling (context of use). This is a very important parameter for the personalization of service (routes) provision. The users categories follow below:

- Tourist (a person in a foreign country or new city that travels for holidays).

- Commuter (a person in his/her city that moves daily to/ from his work).

- Businessman in a foreign country or new city.

- Recreational traveler in his/her city.

- Emergency traveler (a person in his/her city that needs to travel urgently, e.g. to go or get someone at the hospital).

The traveler has different needs if, for example, the reason for travelling is recreation or work, and if he is in his/her town or in a different town/ country. Thus, an effective system should be able to change accordingly when the user type changes, for the same person/user, as the needs for information vary significantly with the reason for transportation.

\section{Algorithm development}

The proposed algorithm presumes that the information to the traveler is provided through mobile devices (phones or PDAs) with different memory capacities and platforms (ranging from Symbian, to iPhones and Android), thus the minimum requirements of the less expensive devices should be taken into consideration. In addition, most likely the timeframe within which the traveler requests information is quite tight (during travelling). For these reasons, only 
the optimal solutions must be given to the user, according to his/her preferences and not just providing him/her a list of possible routes and asking him/her to select. To solve the above problem, an algorithm has been developed in a $\mathrm{PhD}$ thesis [6], with the aim to prioritise the available routes according to the personal profile of each user.

The algorithm is developed in six steps that are described below:

STEP 1 Checking of number of times the user has used the application

The application of the personalisation algorithm is initiated when the user has used the application several $(\mathrm{X})$ times, so that the algorithm can record his/her preferences (in order that the history of his/ her previous selections is formed) and create his/her profile. This is when the maximum acceptable walking distance, the least acceptable transportation mean type and the maximum number of acceptable interchanges will have a value, in accordance to the past user selections. This value $\mathrm{X}$ can be specified by each service provider, however here, it is set as default to 7; this value was defined as a result of comparative user testing [6].

Only when the user has used the application and selected routes seven times or more, the algorithm is ready to continue to the next steps.

STEP 2 Searching of routes satisfying the 3 basic criteria

The algorithm checks if the available routes for the specified points of origin-destination satisfy the maximum acceptable walking distance between PT means, the acceptable transportation mean type and the maximum number of acceptable interchanges. If the sum of available routes found is less than the maximum number of routes that can be shown to the user's device (this is especially important for Symbian mobile phones, with screen size, memory and processing limitations) and the maximum number of routes that can be stored in a back-up list, then the algorithm moves to Step 3.

The prioritization order is based on the following concept:

First, the user will be shown the optimal routes according to the least walking distance, then according to the acceptable transportation means and finally, based on the least requested changes of PT means.

STEP 3 Searching and inclusion of routes that satisfy the criterion of the maximum acceptable walking distance

If there are still free places on the screen capacity of the mobile device or the back-up list, then the routes that satisfy the criterion of the maximum acceptable walking distance are also checked, even if they don't satisfy the rest two criteria. This is done in order to fill the empty places in the user's mobile device.

STEP 4 Searching and inclusion of routes that satisfy the criterion of the acceptable PT mean type

If with the 2 nd and 3 rd step the proposed routes are less than the capacity of the mobile device screen, as well as of the back-up list, the application searches for routes that satisfy the criterion of the least acceptable transportation mean type, even if they don't satisfy the rest two criteria. This is done in order to fill the empty places in the user's mobile device.

STEP 5 Searching and inclusion of routes that satisfy the criterion of the maximum acceptable changes of PT means

If with the 2nd, 3rd and 4th steps the proposed routes are still less than the capacity of the main and secondary solution lists, then the application searches for routes that satisfy the criterion of the maximum acceptable changes of transportation means, even if they don't satisfy the rest two criteria.

In case a route does not satisfy the above rules (steps 2-5), it will be stored on a secondary (backup) list, which will be available only if the user selects to see additional routes or in case there is no other, better solution that can be proposed by the system.

STEP 6 Presentation of routes when the times that the traveler has used the application is less than the set threshold X (History $<\mathrm{X})$

If a particular user has used the system less times than the predefined limit of $X$ times, then the proposed routes that will be presented will be those with the shortest walking distance between interchanges and/or with the acceptable (by the user) transportation means and/or with the least number of interchanges (with this prioritization order). The rest routes will be stored and the user will be able to see them anytime.

During learning of user preferences (History $<\mathrm{X}$ ) by the system, the walking distance, the type and number of transportation means are monitored and the corresponding values/parameters are stored based on the selected route, each time the user makes use of the system. Of course, those three parameters are recorded only when there are at least two alternative routes available, since if the system returns only one proposed route with a walking distance of $\mathrm{x}$ meters, requiring y numbers of interchanges, then the user will have to select it in any case, as it is the only one available in order 
to reach his/her destination. But this selection does not imply that the user prefers to walk up to $\mathrm{x}$ meters, nor that he/she prefers to change up to $y$ transportation means.

If the user selects even once (from the back-up routes) a route that does not satisfy the best routes criteria (i.e. the maximum acceptable walking distance per mode interchange, the acceptable transportation mean types and the maximum acceptable number of transportation means to be used in a route), then this route will be moved to the list of the prioritized proposed routes. Finally, by selecting the unexpected route (in the back-up list) for several times, ${ }^{1}$ the user's profile will be updated and a new learning of the user will be initiated (all the values of the key parameters will be set to 0 ) until History $=\mathrm{X}$. In the meantime (while History $<\mathrm{X}$ ), the routes will be proposed according to the old algorithm.

Of course, if for example a route with more transportation means is in the main list, the route with less transportation means will remain also in the primary list. The same holds true also for the type and number of PT means.

Further personalization: When the best routes are defined according to the above algorithm, there are two filters that are used for further personalization and prioritization, based on the cost and the duration of each route. Such a personalization is applied if the routes that satisfy the requested characteristics exceed the capacity of the main and backup routes lists and the parameters of the maximum acceptable walking distance, the acceptable transportation means types and the maximum acceptable number of transportation means to be used in a route are equally satisfied and that's why prioritization cannot be given.

\subsection{Example of algorithm application}

It is assumed that:

- History $>7$ (the user has used the system more than seven times).

- The mobile device screen is able to show up to five routes in the main list and store another five in the back-up list.

- The user determines his origin point. The system finds 12 alternative routes, with the characteristics as shown in Table 1:

The user profile has been created as follows (according to his/her previous selections):

\footnotetext{
${ }^{1}$ At least $10 \%$ of the ratio of selection based on the new characteristic over the sum of selections since the application use, i.e. (number of selected routes with the new characteristic / number of selected routes by the user since he/she started using the system) $>0.1$.
}

- Maximum acceptable walking distance: $2 \mathrm{~km}$.

- Transportation mean that the user does not wish to use at all: tram.

- Maximum acceptable number of interchanges of PT means: 2.

- Also, the number of selected routes by the user based on the lowest cost are 6 , while the number of selected routes by the user based on the smallest duration are 12 .

Thus, the routes that satisfy the user's profile are 5, namely routes: $1,3,5,6,8$.

After prioritization, the order of the routes will be shown to the user as below:

1. Route 8

2. Route 5

3. Route 6

4. Route 3

5. Route 1

Those five routes will be included in the main list, while the rest in the back-up list.

Since there are five more empty places in the back-up list, the algorithm checks the rest routes that satisfy the criterion of the maximum acceptable walking distance, which are two, namely routes no. 2 and 7.

The order with which those two routes will be presented at the user, is:

6. Route 7

7. Route 2

There are still three empty places left in the back-up list, thus the algorithm checks the rest routes that satisfy the criterion of the acceptable PT means. There is only one such route, which is route number 10 .

This means that one more route will be added in the additional/secondary list:

\section{Route 10}

Finally, in order to fill up the remaining two empty places in the back-up list, the algorithm examines the rest routes that satisfy the criterion of the maximum acceptable PT means interchanges, and finds that there are three, namely routes no. 4, 11 and 12 .

The algorithm will prioritise the last three routes according to the less PT means interchanges, since there is no room for one route (only two more routes can be added in the additional list). But since all three routes have the same number of transportation means (i.e. 1), further personalisation will be applied, based on the duration and the cost of each route. 
Table 1 Alternative routes characteristics

\begin{tabular}{|c|c|c|c|c|c|}
\hline Route number & $\begin{array}{l}\text { Maximum walking } \\
\text { distance per PT mean }\end{array}$ & $\begin{array}{l}\text { Acceptable PT } \\
\text { means to be used }\end{array}$ & $\begin{array}{l}\text { Maximum acceptable } \\
\text { PT means interchanges }\end{array}$ & Cost & Duration (minutes) \\
\hline 1 & 2 & $\sqrt{ }$ & 2 & & 14 \\
\hline 2 & 2 & $\mathrm{x}$ & 2 & & 12.5 \\
\hline 3 & 1.9 & $\sqrt{ }$ & 2 & & 12 \\
\hline 4 & 2.5 & $\mathrm{x}$ & 1 & $7 €$ & 10 \\
\hline 5 & 1 & $\sqrt{ }$ & 1 & & 10 \\
\hline 6 & 1.8 & $\sqrt{ }$ & 1 & & 9 \\
\hline 7 & 1.5 & $\mathrm{x}$ & 1 & & 7 \\
\hline 8 & 0.5 & $\sqrt{ }$ & 2 & & 13 \\
\hline 9 & 3 & $\mathrm{x}$ & 4 & & 18 \\
\hline 10 & 2.4 & $\sqrt{ }$ & 3 & & 15 \\
\hline 11 & 3 & $\mathrm{x}$ & 1 & $8 €$ & 11.5 \\
\hline 12 & 2.3 & $\mathrm{x}$ & 1 & $6.5 €$ & 9 \\
\hline
\end{tabular}

Thus, the quickest routes will be added in the secondary list:

9. Route 12

10. Route 4

In conclusion, route no. 11 will not be shown at the user at all. The same holds true for route no. 9, as it does not satisfy any of the above criteria.

\section{Evaluation and results}

The algorithm described above was implemented to run on a mobile device platform, in order to be tested by real users. Intelligent agents were used for the development. Also, a PDA emulator was developed, simulating the application functionality with the PDA on the PC.

The above-described algorithm was evaluated with ten users. In order to limit the occupation time of each user, the threshold for History was set to 7 (i.e. each user had to use the application 7 times $(X=7)$ in order to build his/her personalization profile).

Six out of ten users tested the application on PDA, while the rest four used the PDA emulator on the PC, due to limited devices. Each user had about $30 \mathrm{~min}$ available to test the application.

\subsection{Participants data}

The users' data was gathered through a questionnaire that was specifically designed for the multimodal route guidance system evaluation. This questionnaire consists of two parts; The first part dealt with generic questions on the age, level of studies and user's experience in using similar applications, while the second part included questions about the application. An equal number of men and women was used.

The average age of the users was 31 years old (min: 25, max: 38). Nine out of ten users had never participated in a testing of a prototype application. It should be noted here that young persons were selected that were acquainted with the use of PCs and mobile devices, in order to be able to assess the functionality and usefulness of the developed application, without being influenced by the possible difficulty in the use of such devices. Seven users declared that they had received transportation related information (e.g. info on public transport, points of interest, etc.) through PCs or mobile devices.

\subsection{Description of the use of the application}

During trials, multimodal routes were requested by the users with all the three criteria (maximum acceptable walking distance, type and number of PT means to be used) and their combinations. In the example that follows below, the routes personalization is shown taking into account the first criterion described in chapter 3 above, i.e. the maximum acceptable walking distance per transportation mean change.

Using the application for the first time, the user receives five alternative routes (Fig. 1, left screenshot), for which he can see the maximum walking distance between intermodal changes (Fig. 1, right screenshot).

During the next uses of the application, the user selected five times the route with $400 \mathrm{~m}$ maximum walking distance and two times the route with $350 \mathrm{~m}$ maximum walking distance. This means that the next time the user will use the system, it will propose routes with maximum walking distance 


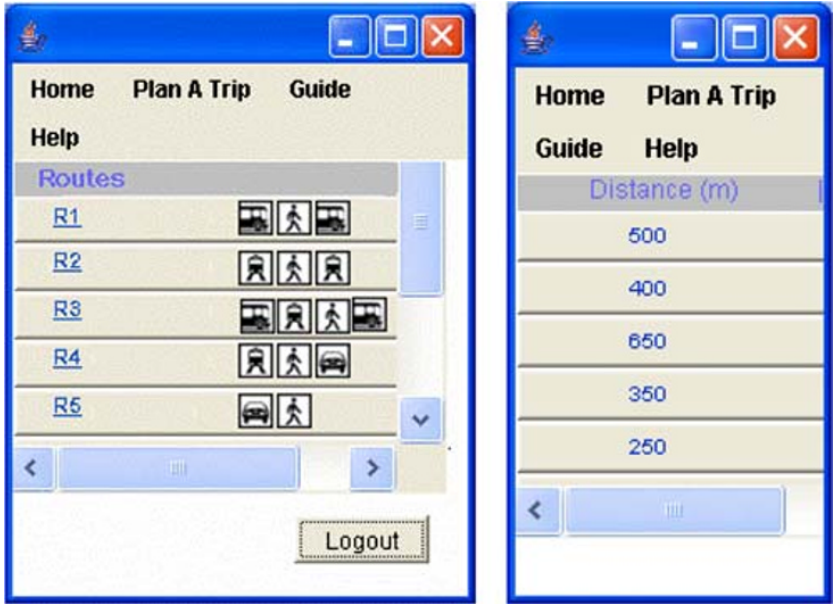

Fig. 1 Proposed routes by the system before personalization

less or equal to $400 \mathrm{~m}$. Indeed, the application returns only three such routes that satisfy this criterion (Fig. 2).

Note that the personalized results retrieved by the application are prioritized, starting with the route with the smallest walking distance on the top, towards those with bigger distances.

\subsection{Results}

The users' acceptance opinion regarding the personalized application in relation to a non-personalised one is depicted in the following diagram. Feedback is provided based on a 5-scale system, from positive to negative (Fig. 3).

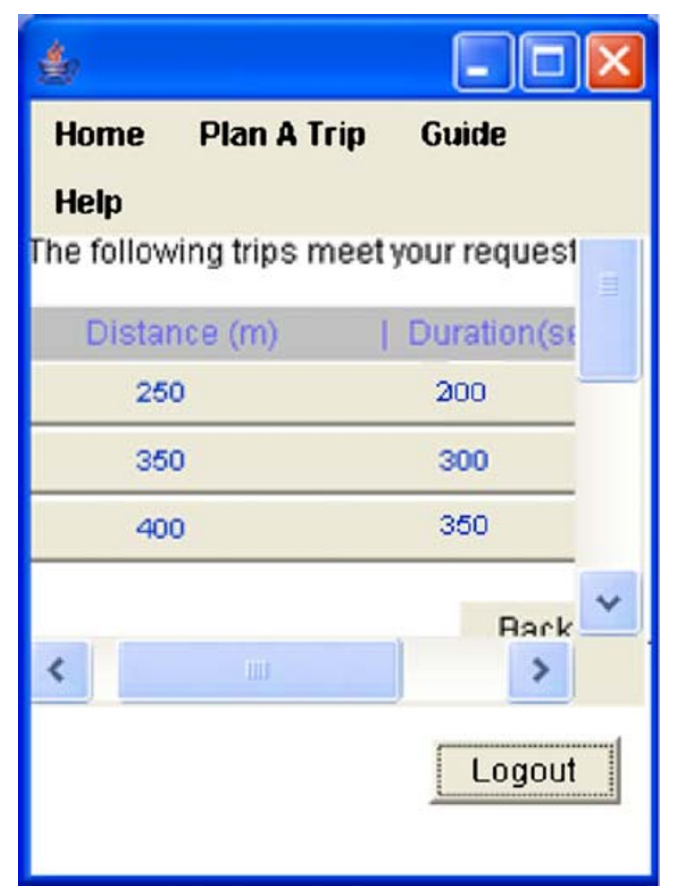

Fig. 2 Proposed routes by the system after user profile creation

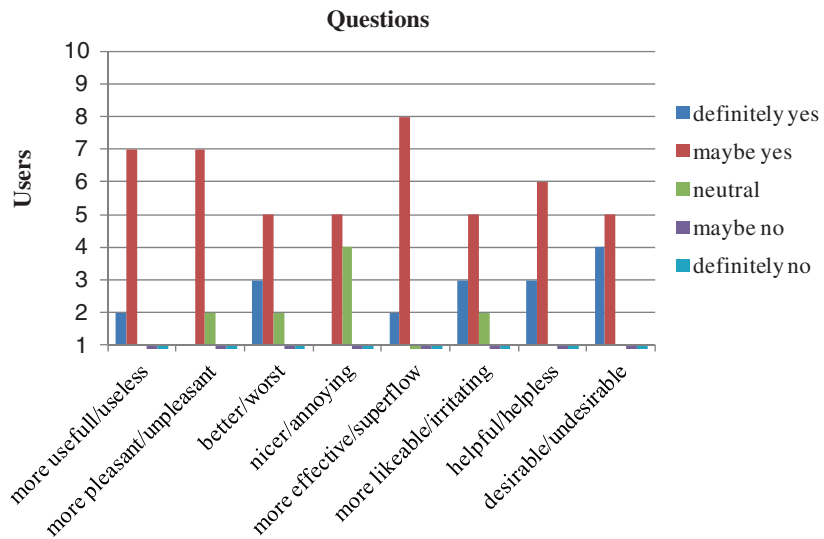

Fig. 3 Results of users' acceptance for the personalised multimodal routes provision application in relation to the non personalized one

According to the above figure, the majority of the users gave a marking ' 2 ' to all the questions, while there are several users that ranked the application with the best mark ' 1 ' and less that gave a mark ' 3 ' (mark that denotes a neutral opinion).

Analyzing the average scoring value of the questions, it can be clearly seen that the users have a positive opinion for the application of personalized routes provision (since all the values are closer to the best possible scoring ' 1 ', rather than to the most negative one). The best scoring ( 1.7 points) has been given to the characterisation that the application is desirable, followed by its effectiveness and helpfulness (i.e. valuable), while the most negative scoring (2.3 points) has been given to the question if it is nice/annoying. Of course, even this scoring is overall positive as it is quite less than the mean/ neutral ranking and far from the most negative possible one $(5$ points) (Fig. 4).

The tests participants were asked to indicate the confidence level for the application, as a \%. The detailed results per user follow next: (Fig. 5)

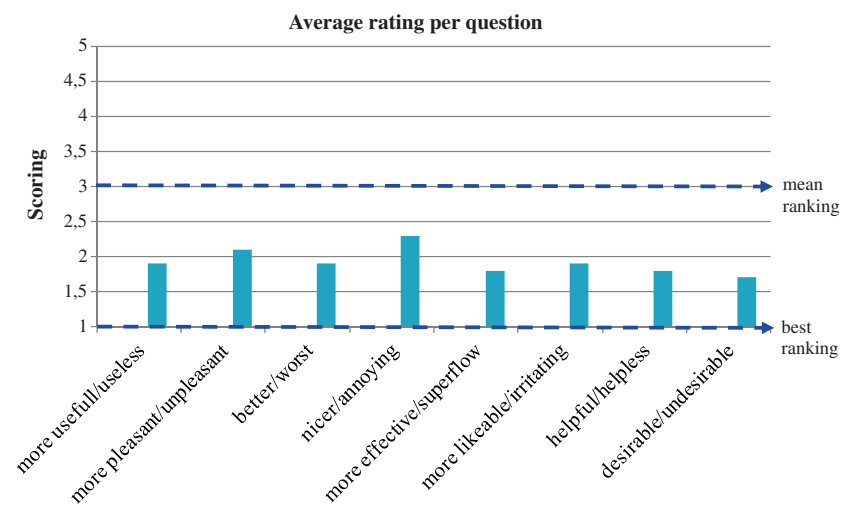

Fig. 4 Mean scoring in relation to the optimal, average and worst scoring, for each question of user acceptance 


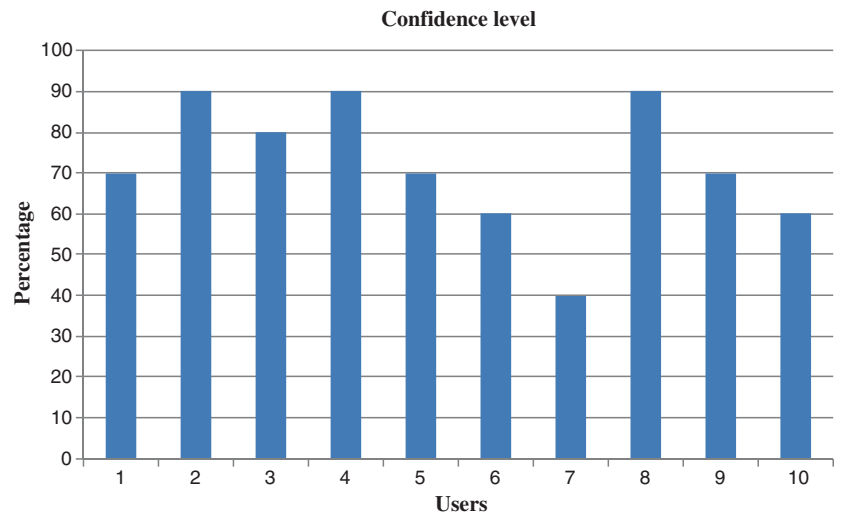

Fig. 5 Confidence level to the personalized application, per user

Since nine out of ten users expressed a confidence above $60 \%$ (reaching $90 \%$ by three users) and the average rating is $72 \%$, it is clear that the results are very encouraging and that nearly all users (apart from user no. 7) indicate that they can trust the application. The standard deviation is 16.19 , showing that there is a significant variation among the users feedback. However, if user 7 is not taken into account, the standard deviation is reduced to 12 , showing a more similar scoring among the participants.

\subsubsection{Importance of personalized parameters}

The tests participants' opinion was captured regarding the significance of the personalisation parameters. They had to select maximum three parameters from the predefined list below or add a new one:

- Selection of transportation mean type

- Maximum walking distance

- Number of interchanges among transportation means

- Minimum transportation time

- Minimum transportation cost

- Other

All the users selected three parameters. The results are shown in Fig. 6.

The maximum walking distance is evidently considered as a crucial parameter by all the users. This parameter is even more important for elderly or disabled travelers. The next important parameters are the number of interchanges and the minimum transportation time. The fact that the users wish to travel fast and comfortably (without many interchanges) is reasonable. In a real service, the importance of these two parameters will change depending on the user type. For instance, if the user is a tourist, the number of interchanges may be more important than the total duration of the trip, and vice versa if the traveler is a businessman.



Fig. 6 Results of the most important personalization parameters for transportation

Still, other factors (e.g. total available time) may influence further their relevant significance.

The selection of the transportation mean (or even better, the exclusion of transportation means) plays an important role for some users, while the total cost seems to be the least important parameter. These two parameters depend on the travelling frequency and the financial status of each traveler.

None of the users proposed any new personalization parameter that should be taken into account, as for example could be the safest or the most picturesque route.

\section{Conclusion}

Specific algorithms have been developed, as part of a $\mathrm{PhD}$ thesis, allowing the traveler to receive personalized information on the multimodal route to follow in order to reach a desired destination. The comparison of the developed application to a non-personalised one by ten users, showed an impressive advantage of the personalized application, which is expected to make it viable and financially exploitable. It is worth mentioning that the personalization used at the tests, was based just on seven previous uses of the application by the tests participants, while an application/service that would take in to account much more and chronically distributed choices of the user, could obtain much higher reliability and usefulness.

The personalization of a service provided through PC may not be as important as in the case of a mobile device because the user has more time and comfort to review all alternative routes. While on the move, personalization is seen as an imminent need for the traveler since the user has time and space limitations to find the optimal route for him/her.

\subsection{Limitations and extensions}

The assessment of the algorithm by the users was very positive, confirming this way the added value of the personalized application/services. However, there are at least two 
parameters that should be taken into account when generalizing the results:

\section{- Sample size}

All the algorithms were tested by ten users ( 5 men and 5 women). This sample is enough for extraction of qualitative conclusion, however it is not statistically significant for quantitative reliable results. Before the wide use of such a system, a large-scale pilot application is required, with many users.

- Long-term personalization consequences

All the users tried both the personalized and the nonpersonalised application for a short time (less than a day). However, the long-term use of a personalized service may entail dangers, such as:

- If the system is personalized once and then it remains as it is, not allowing continuous dynamic re-processing of the thresholds, the change of the user's preferences may result that the system is nonoptimal for him/her anymore (e.g. the user does not wishes to have many interchanges).

- If the system allows the continuous dynamic reprocessing of the personalization thresholds, it may be considered by the user as non-stable leading to dissatisfaction, since he/she receives 'different routes for the same request'. Such an effect could question the service confidence level.

For this reason, the optimal application of the personalisation algorithms to a real service, implies studies of longterm consequences, where the system will be used by a big number of users for 6-12 months.

There are several other applications that could make use of the proposed algorithm, namely:

- Adaptation of the presentation means (user interface) of information to the user, depending on the user disabilities, in order to automatically offer accessible information for all users.

- The anonymous gathering of the user's preferences and their statistical analysis by the relevant service providers may lead to the improvement of relevant services, e.g. assignment of PT means to alternative routes, times, etc.

- Several environmental parameters could be taken into account for the information provision, as the weather conditions, time and financial limitations, etc. Optimally, with the use of stochastic methods (e.g. neural networks) the personal algorithm of route selection for each traveller could be reached. Thus, the user instead of choosing the fastest, shortest, safest, more accessible or more picturesque route, he/she will just need to choose the most 'desirable for him/her' one, taking into account a combination of personal and environmental parameters.

- The way of information presentation and its content should consider the transportation mean where the user is on, as well as his/her workload. Technically, it has not been solved yet and it certainly constitutes an interesting research area. For example, when the user is on the metro, or even the bus, it is possible to lose the satellite signal (GPS) and a WiFi, 3 G, etc. connection might not be available. Then, his/her localisation should be achieved with other means (e.g. use of GSM triangulation, 'logical' positioning, hybrid positioning, etc.), which haven't yet obtained the required reliability and fidelity levels. Similarly, it is very difficult for the service to know the current workload of a traveler (e.g. when he/she is purchasing tickets).

Finally, as the sample is fairly small (as already mentioned above), further research is needed to pair the qualitative evaluation through questionnaires with a more quantitative analysis of the traveler usage of the application. The current paper provides a sufficient proof of concept of the suggested methodologies, nevertheless results need to be checked against a bigger sample size in further research.

Open Access This article is distributed under the terms of the Creative Commons Attribution License which permits any use, distribution and reproduction in any medium, provided the original author(s) and source are credited.

\section{References}

1. Editors Desk (2009) Mobile web: latest facts and stats forecast a rosy outlook

2. Gartner Newsroom (2011) Gartner identifies 10 consumer mobile applications to watch in 2012

3. James A (2010) (http://www.gsmdome.com/marketing/5-billionmobile-services-users-worldwide-reached-according-to-ericsson_17223)

4. Mizaras V, Manos T, Pachinis T, Batsis A, Beck P, Weisser J, Pretsch T, Petraki E, Spanoudakis N, IM@GINE IT Deliverable 1.1 (2005) Use cases and user/vehicle profile requirements

5. Mobile Life: GTI (2011) (http://discovermobilelife.com/files/The $\%$ 20Status\%20Quo\%20-\%20The\%20Role $\% 20$ of $\% 20$ Mobility $\% 20$ in $\% 20$ Consumer\%20Lives.pdf)

6. Panou M (2008) PhD Thesis "An advanced, personalized travelers information and warning system", Aristotle University of Thessaloniki

7. Simões A, Gomes A, Bekiaris E, ASK-IT (IST-511298) Deliverable 1.1.2 (2006) Use cases

8. TNS Mobile Life, GTI (2011) (http://audio4cast.com/2011/05/ page/2/)

9. World Travel Trends Report (2010/2011) (http://www.itb-berlin.de/ media/itb/itb_media/itb_pdf/worldttr_2010_2011 1.pdf) 